\title{
Complete Atrioventricular Block Complicated with Cardiogenic Shock as Primary Manifestation of a Novel Coronavirus Infection in a Child: A Rare Case Report
}

\author{
Feisal Rahimpour ${ }^{1}$, Mohsen Yaghubi ${ }^{2}$, Reza Ghasemi ${ }^{3}$ and Mahmood Hosseinzadeh Maleki ${ }^{4}$ \\ ${ }^{1}$ Assistant Professor, Department of Pediatric Cardiology, Imam Reza Hospital, Mashhad University of Medical Sciences, Mashhad, Iran \\ 2Department of Extra-Corporeal Circulation (ECC), Razavi Hospital, Imam Reza International University, Mashhad, Iran \\ ${ }^{3}$ Assistant Professor, Department of Cardiology, 9 Dey Educational Hospital, Torbat Heydariyeh University of Medical Sciences, Torbat Heydariyeh, Iran \\ ${ }^{4}$ Associated professor, Department of Cardiac Surgery, Imam Reza hospital, Mashhad University of Medical Sciences, Mashhad, Iran
}

* Corresponding author: Mahmood Hosseinzadeh Maleki, Department of Cardiac Surgery, Imam Reza hospital, Mashhad University of Medical Sciences, Mashhad, Iran. Email: mahmoodhosseinzadeh@yahoo.com

Received 2021 January 24; Revised 2021 April 11; Accepted 2021 April 17.

\begin{abstract}
Background: Since late 2019, the outbreak of Coronavirus Disease -2019 (COVID-19) has rapidly spread worldwide. Due to the novelty of this unknown disease, many of its manifestations are in an aura of ambiguity. Early data showed that children diagnosed with this virus had severe or critical situations combined with cardiovascular complications, such as myocardial injury, heart failure, ventricular dysfunction, shock, and even Kawasaki-like disease. We describe a cardiac involvement of this disease that presented with a complete atrioventricular block in a child.

Case presentation: A 4-year-old boy patient was admitted to the emergency department with a seizure and decreased consciousness level. Moreover, he was in a febrile state and a decreased level of oxygen saturation. Based on his critical situation, he was transferred to Pediatric Intensive Care Unit (PICU) and prepared to initiate invasive mechanical ventilation. High-resolution computed tomography revealed dependent significant grand gloss opacities with interlobular septal thickening in both lungs. The Polymerase chain reaction test confirmed "severe acute respiratory syndrome coronavirus 2 (SARS-CoV-2)". An Electrocardiography showed a complete atrioventricular block pattern. According to the cardiac involvement, we prescribed a specific medication protocol. After 80 hours of PICU entrance and eight days after it, he was weaned from mechanical ventilation and discharged in good condition from the hospital.

Conclusion: It shows that rapid diagnosis of cardiac conduction system involvement of SARS-COVID-19 infection in children can enhance the prognosis. Furthermore, applying an optimal strategy for the treatment leads to effective therapy.
\end{abstract}

Keywords: Cardiogenic shock, Electrocardiography, Heart block, SARS-CoV-2

\section{Background}

Since late 2019, the main presentation of the novel coronavirus disease 2019 presents respiratory system impairment in our evolving knowledge. Some reports are suggesting that SARS-CoV-2 can affect many organs and systems such as the cardiovascular system and its complications are another critical aspect of the disease that might cause severe and irreversible damage $(1,2)$.

Herein, we report a pediatric case with a novel coronavirus with cardiac damage, which involved the conduction system.

\section{Case Presentation}

A 4-year-old boy patient weighing $17 \mathrm{~kg}$ was admitted to the emergency department with a seizure and altered level of consciousness. Before admission, he had been suffering from an upper respiratory infection for at least ten days and the symptoms had worsened in the past two days. He had no family history of neurological disorders, cardiovascular disease, sudden cardiac death, or syncope.

On admission, hemodynamic parameters were in the critical range. He had a blood pressure of 86/69 $\mathrm{mmHg}$, heart rate of 54 beats/minute, and respiratory rate of 39 breaths/minute, and had a fever with a tympanic temperature of $38.9^{\circ} \mathrm{C}$. Oxygen saturation was $90 \%$ at rest with a finger pulse oximeter on the index finger. Primary physical examination was also performed, and the bilateral crackles were found in the upper and lower of the chest in auscultation.

Due to his critical situation, the patient was immediately transferred to the Pediatric Intensive Care Unit (PICU).

The arterial blood gas (ABG) sampling was instantly done. ABG indicated severe acute respiratory acidosis with $\mathrm{PH}=7.05, \mathrm{PaCO} 2=76$, and HCO3 $=28$. The oxygen saturation of the ABG sample was $65 \%$, and also $\mathrm{PaO} 2$ was $34 \mathrm{mmHg}$. Five minutes later, he was prepared to initiate invasive mechanical ventilation due to respiratory failure, and based on the disrupted ABGs. Accordingly, endotracheal intubation was done. Mechanical ventilation mode was performed with Volume controlledSynchronized Intermittent Mandatory Ventilation [VC-SIMV]. Besides, fentanyl infusion (10-50 mcg/h) was prescribed by an anesthesiologist. 
The portable anteroposterior chest radiography was emergently done which indicated significant diffused bilateral infiltration consistent with severe viral pneumonia. The chest high-resolution computed tomography (HRCT) was taken without using contrast as another diagnostic imaging test. The results revealed dependent significant grand gloss opacities with interlobular septal thickening in both lungs.

The laboratory evaluation indicated an elevated white blood cell count $(19250$ cells $/ \mathrm{ml})$ with neutrophilic predominanceand lymphopenia (7250 cells/ul). Other laboratory findings revealed elevated high-sensitivity troponin $(1.07 \mathrm{ng} / \mathrm{ml})$; elevated CPKMB level (133 g/L), brain natriuretic peptide $(2,000$ $\mathrm{pg} / \mathrm{ml}$ ). Inflammatory markers such as C-reactive protein $(22 \mathrm{mg} / \mathrm{dl})$, ferritin $(1,138 \mathrm{ng} / \mathrm{ml})$, and $\mathrm{D}$ dimer, $3.1 \mathrm{mg} / \mathrm{ml}$, was elevated.

Due to symptoms of infection of SARS-CoV-2 during the outbreak period, the PCR taken from his upper respiratory tract confirmed the diagnosis of acute respiratory syndrome coronavirus 2 (SARS-CoV-2).

An electrocardiogram (ECG) was done during the first hours of admission to the ICU, which showed a complete pattern of an atrioventricular block (CAVB) (Figure 1).

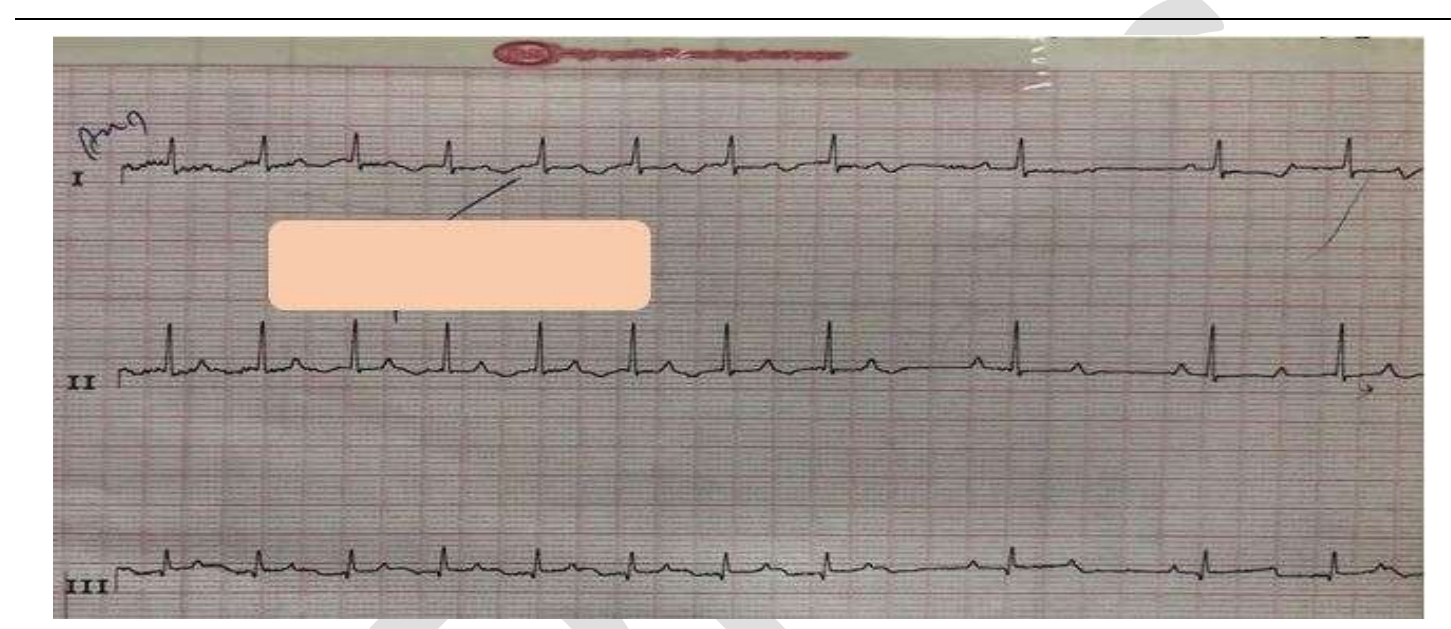

Figure 1. Three-limb lead electrocardiogram (ECG) of the patient after ICU admission revealed a complete atrioventricular block. Secondary ST-T change is also present.

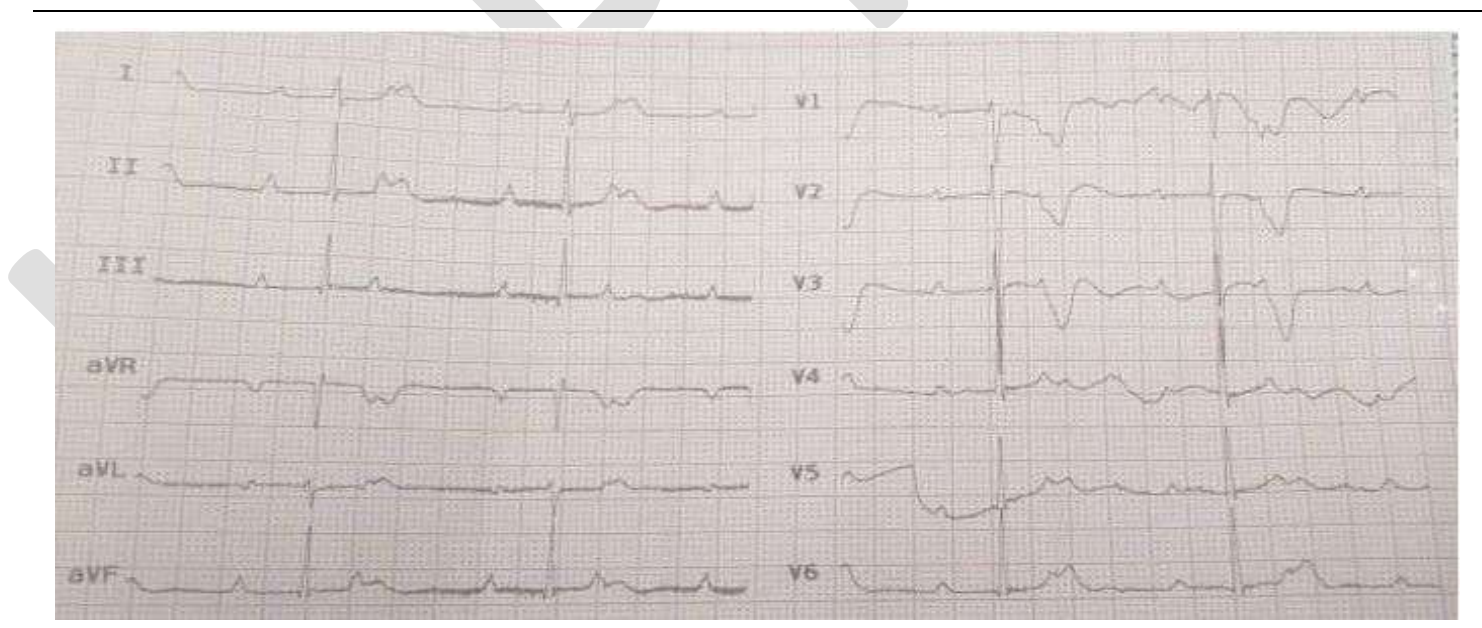

Figure 2. 12-lead electrocardiogram (ECG) of the patient five days after ICU admission. There are normal axis deviation and 2:1 atrioventricular block (Mobitz type 2). Secondary ST-T change is also present.

Transthoracic echocardiography was done and revealed reduced ejection fraction (about 30\%; eye estimation) along with diffuse myocardial dyskinesia. Another complication, such as pericardial effusion, was rejected. No evidence of significant valvular heart disease was found.

With these regards, we decided to prescribed the intravenous immunoglobulin (IVIG); $500 \mathrm{mg} / \mathrm{kg} /$ day, dexamethasone; $\quad 0.15 \mathrm{mg} / \mathrm{kg} / \mathrm{QID}, \quad$ milrinone;
$0.5 \mathrm{ug} / \mathrm{kg} / \mathrm{min}$, and dopamine; $5 \mathrm{ug} / \mathrm{kg} / \mathrm{min}$ based on the involvement of the patient with COVID-19.

After three days in ICU, the ventilatory and ABG parameters were within an acceptable range. Therefore, a weaning plan was established. The patient weaned, and extubation was thoroughly performed 2 hours later 80 hours after mechanical ventilation. He was closely monitored and treated with supplemental oxygen therapy using high-flow 
nasal at $40 \mathrm{Lit} / \mathrm{min}$ and $\mathrm{FiO} 2=40 \%$.

Additionally, the level of troponin I was significantly reduced to $0.3 \mathrm{~g} / \mathrm{L}$. Moreover, the echocardiographic parameters showed that the LVEF of the patient gradually recovered to $50 \%$.

After five days of stay in ICU, the inotropes were tapered and change to captopril $(1 \mathrm{mg} / \mathrm{kg} /$ day) and furosemide $(1 \mathrm{mg} / \mathrm{kg} /$ day). The ECG pattern changed from CAVB to Second-degree Atrioventricular block (2:1), and the hemodynamic was stable (Figure 2).
On the sixth day, the patient was treated by supplemental oxygen therapy using a simple face mask followed by a nasal cannula with a flow of 3 lit/min. The patient with an excellent general condition was transferred to the post-ICU. Two days later, he was discharged and advised his parents to follow the cardiac arrhythmia at least two months later.

After two months, the follow-up ECG revealed a persistent Second-degree Atrioventricular block (2:1)

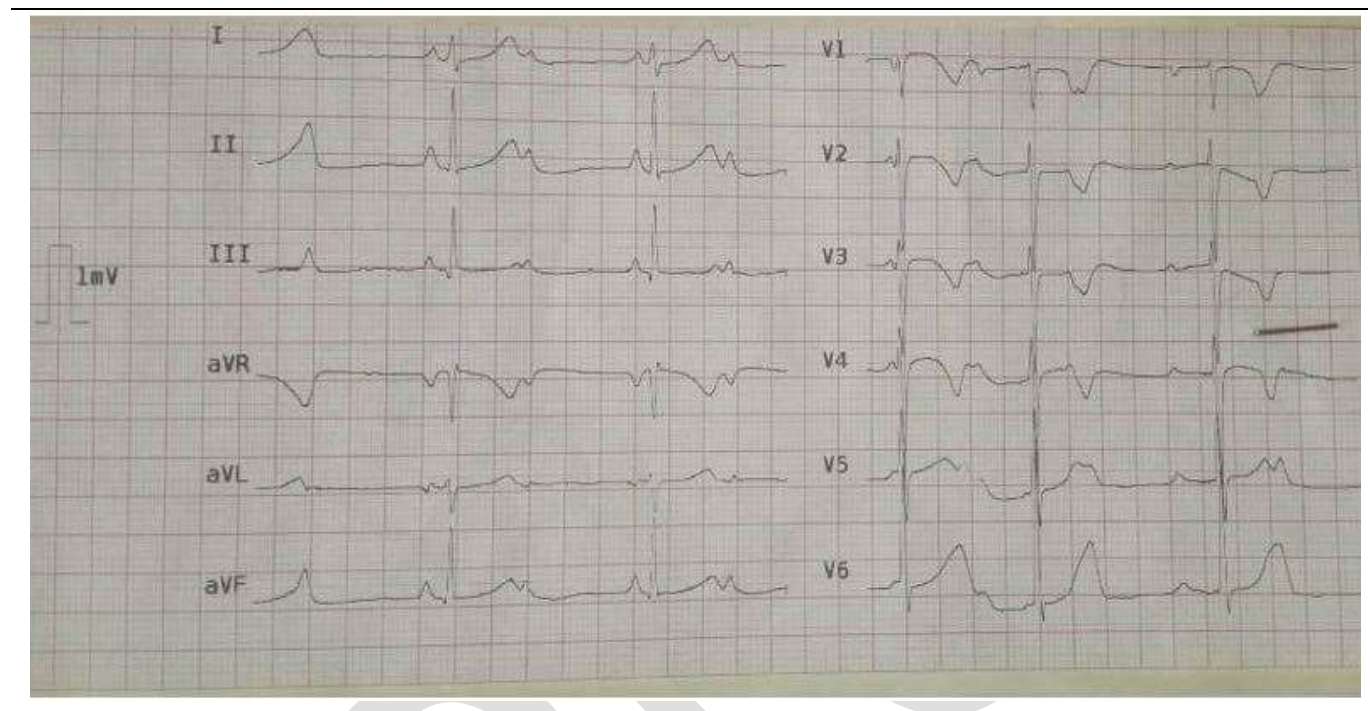

Figure 3. 12-lead electrocardiogram (ECG) of the patient two months after ICU discharge. There is a persistent 2:1 atrioventricular block (Mobitz type 2). Also, secondary ST-T changes were observed.

(Figure 3). Also, trans-thoracic echocardiography showed normal wall motion without evidence of dyskinesia, and LVEF was estimated at 55\%.

Finally, persistent dysrhythmia can lead to fatal dysrhythmias; the patient was monitored by Holter and recommended his parents to continue the followup treatment with an electrophysiologist.

\section{Discussion}

Cardiovascular involvement with the novel coronavirus (COVID-19), ranging from mild to severe presentation with prognosis in acute cardiac injuries (3).

Meanwhile, some studies have reported that pediatrics with COVID-19 tend to show mild cardiac dysfunction compared to adults $(4,5)$.

This fact is not consistent with clinical and paraclinical findings of our patient presented with severe secondary cardiac complication due to SARS-CoV-2 infection. It is noteworthy that the coincidence of severe myocardial injury and conduction system impairment in pediatrics was first reported in this case study.

The mechanism of COVID-19-associated myocardial injury and shock is unclear. It may be due to cytokine-mediated myocardial inflammation $(6,7)$.

Furthermore, based on endomyocardial biopsy, cardiac injury in patients with novel coronavirus may be due to direct viral infection causing myocarditis (8).

However, the mechanism for COVID-19-induced heart block is not well studied. We suspect that it is secondary to inflammation of the conduction pathways as a result of the direct viral invasion and immune-mediated injury $(9,10)$.

Also, the use of intravenous immune globulin (IVIG) combined with a systemic glucocorticoid may attenuate inflammatory response in patients with COVID-19 and myocarditis (11).

In our case, the patient had significantly elevated inflammatory markers. This first-line drug causes our patient to respond positively to conduction impairment and inflammation by resolving CAVB which suggests that inflammation is probably the leading cause of conduction disease in our patient. This protocol can be established as an effective treatment since there is no definite treatment recommendation for concomitant myocardial injury and inflammation of conduction pathways that may be completely coincidental.

Despite the reverse of CAVB to atrioventricular block with the treatment of the patient, this abnormality of conduction persists for a long time without any change. Therefore these patients need long-term follow-up electrophysiologic study to prevent life-threatening arrhythmias. 


\section{Conclusion}

Nevertheless, the fact that COVID-19 can cause myocarditis and, consequently, conduction disturbances lead to pediatric cardiogenic shock. Therefore, these features of cardiac involvement of COVID-19 in pediatrics should be considered as emergent situations.

\section{Acknowledgments}

The authors would like to express their sincere gratitude to the nurses of pediatric cardiology of Mashhad University of medical sciences and patients participating in the research.

\section{Footnotes}

Conflicts of Interest:The authors declare that there is no conflict of interest regarding the publication of the present study.

Declaration of Patient Consent: The authors certify that they have obtained appropriate patient consent forms. The families have agreed to have their pictures and other clinical information reported in the journal. They were also assured that their names and initials would not be published and efforts would be made to conceal their identities, but anonymity could not be guaranteed.

\section{References}

1. Xiong TY, Redwood S, Prendergast B, Chen M. Coronaviruses, and the cardiovascular system: acute and long-term implications. Eur Heart J. 2020;41:1798-800. doi: 10.1093/eurheartj/ehaa231. [PubMed: 32186331].

2. Sadeghipour P, Talasaz AH, Eslami V, Geraiely B, Vojdanparast
M, Sedaghat $M$, et al. Management of ST-segment-elevation myocardial infarction during the coronavirus disease 2019 (COVID-19) outbreak: Iranian"247" National Committee's position paper on primary percutaneous coronary intervention. Catheter Cardiovasc Interv. 2020;97(3):E346-51. doi: 10.1002/ccd.28889. [PubMed: 32320138].

3. Li JW, Han TW, Woodward M, Anderson CS, Zhou H, Chen YD, et al. The impact of 2019 novel coronavirus on heart injury: a systematic review and meta-analysis. Prog Cardiovasc Dis. 2020;63(4):518-24. doi: 10.1016/j.pcad.2020.04.008. [PubMed: 32305557].

4. Castagnoli R, Votto M, Licari A, Brambilla I, Bruno R, Perlini S, et al. Severe acute respiratory syndrome coronavirus 2 (SARSCoV-2) infection in children and adolescents: a systematic review. JAMA Pediatr. 2020;174(9):882-9. doi: 10.1001/jamapediatrics.2020.1467. [PubMed: 32320004].

5. Dong Y, Mo X, Hu Y, Qi X, Jiang F, Jiang Z, et al. Epidemiology of COVID-19 among children in China. Pediatrics. 2020;145(6):e20200702. doi: 10.1542/peds.2020-0702. [PubMed: 32179660].

6. Akhmerov A, Marban E. COVID-19 and the heart. Circ Res. 2020;126(10):1443-55. doi: 10.1161/CIRCRESAHA.120.317055. [PubMed: 32252591].

7. Li H, Liu L, Zhang D, Xu J, Dai H, Tang N, et al. SARS-CoV-2 and viral sepsis: observations and hypotheses. Lancet. 2020;395(10235):1517-20. doi: 10.1016/S01406736(20)30920-X. [PubMed: 32311318].

8. Tavazzi, G, Pellegrini, C, Maurelli, M, Belliato, M, Sciutti, F, Bottazzi, A, et al. Myocardial localization of coronavirus in COVID-19 cardiogenic shock. Eur J Heart Fail. 2020;22(5):9115. doi: 10.1002/ejhf.1828. [PubMed: 32275347].

9. Liu PP, Blet A, Smyth D, Li H. The science underlying COVID-19: implications for the cardiovascular system. Circulation. 2020;142(1):68-78. doi: 10.1161/CIRCULATIONAHA.120.047549. [PubMed: 32293910].

10. Yaghubi M, Dinpanah H, Ghanei-Motlagh F, Kakhki S, Ghasemi R. Trifascicular block as primary presentation of the cardiac amyloidosis; A rare case report. ARYA Atheroscler. 2018;14(2):101-4. doi: 10.22122/arya.v14i2.1676. [PubMed: 30108642].

11. Hu H, Ma F, Wei X, Fang Y. Corrigendum to: Coronavirus fulminant myocarditis saved with glucocorticoid and human immunoglobulin. Eur Heart J. 2021;42(2):191. doi: 10.1093/eurheartj/ehaa248. [PubMed: 32236443]. 